\title{
Enhanced Physical Document Management using NFC with Verification for Security and Privacy
}

\author{
Z. Zainal Abidin ${ }^{1}$, N.A. Zakaria ${ }^{2}$, Z. Abal Abas ${ }^{3}$, A.A. Anuar ${ }^{4}$, N. Harum ${ }^{5}$, M.R. Baharon ${ }^{6}$, Z. Ayop ${ }^{7}$ \\ Information Security Forensics and Computer Networking \\ Universiti Teknikal Malaysia Melaka (UTeM), Melaka, Malaysia ${ }^{1,2,4,5,6,7}$ \\ Optimization Modelling Analytic and Simulation, Universiti Teknikal Malaysia Melaka (UTeM), Melaka, Malaysia ${ }^{3}$
}

\begin{abstract}
This study focuses on implementation of physical document management for an organization using Near-Field Communication (NFC) since it provides faster detection on tracking items based on location. Current physical document management operates using bar-codes. However, barcodes are able to be duplicated, which make it not secure that lead to forgery and unauthorized modification. Therefore, the purpose of the proposed physical document management system is to produce a better administration control in an organization through the use of verification mechanism. Nonetheless, the current NFC based system is lack of verification process. Thus, an enhancement in physical document management with verification process is proposed and self-developed system is built using C\#, SQLite, Visual Studio, NFC tag and NFC reader (ACR122U-A9). Moreover, the new system required employee to log in to the system by scanning ID tag and followed by the physical document File tag, which both tags scanned at the NFC reader. Then, information of the physical file coordinator and the status of the location of the physical document file is displayed. The significant of this study is to protect confidential document and improve administrative control through dual verification; and produce a database to monitor the real-time data detection.
\end{abstract}

Keywords-Document file management system; physical document files detection; near-field communication (NFC)

\section{INTRODUCTION}

Physical document management system contains a collection of hardcopy documents that has been an important record and evidence in most organizations. The printed document may consist of high classified document or need a right of permission to distribute [1]. The security of the classified hardcopy documents need a systematic filling system and safe implementation [2] due to high reliability on most working procedures. In fact, the crucial hardcopy document must be protected from threats and attacks such as forgery [3], falsification and unauthorized modification.

With the aid of technology adapted into the physical document management system, the integration of autonomous system contributes to faster access to information, more efficient, safe environment and convenient for user [4] in obtaining the printed version of document for further process.

Moreover, a significant change in technological, social and economic system is happening and would be evolved due to industrial revolution [5]. The change in industrial revolution is evaluated with the consequences method for measuring the indicator performance in the implementation phase that aligned with the 21 st century. [6], [7].

Thus, this paper proposes an verification as one of the security feature, which making the revolution of industry 4.0 as a reality. The development phase is done at the document and employee databases. The employee database is linked together with document database in searching for a "match" information between employee who holds the document file and the NFC tag that contain information of the physical document. The new method reduces the time consumed in searching the document file and increase the satisfaction when the employee who wants to find the current position of the physical file at instance.

Additionally, the matching method with verification helps the document management system to be more efficient, effective, avoid human error and reduce time for searching the document folder at the document room. In fact, the system is convenient as the employee needs to tap his or her ID for verification at the NFC reader and scan the document file's NFC tag for searching the "match" data.

In this study, an experiment has been implemented for detecting the availability of document in the folder, folder's name and the employee in-charge who holds the folder of the document at the current location.

Thus, this paper introduces the implementation of physical document detection using NFC for physical document management system. Each of the existing studies on technology used for physical document management is introduced in section 2.0, the related work. The barcode, RFID and NFC is explained in sections $\mathrm{A}, \mathrm{B}$ and $\mathrm{C}$.

\section{RELATED WORK}

\section{A. Barcode}

Barcode is a machine-readable, data representation in the form of bars and spaces on a surface. Furthermore, barcode is an identification code that contains a well-defined combination of parallel lines [8]. Barcode is useful for automatic identification using endpoint devices such as Point of Sales $(\mathrm{PoS})$ as shown as in Fig. 1.

A Special thanks to Malaysia Research Assessment (MyRA) and Universiti Teknikal Malaysia Melaka (UTeM) for giving a sponsor. 


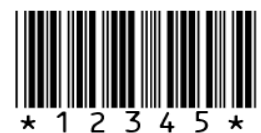

Fig. 1. Barcode [9].

A barcode is machine-readable optical label that can be captured by the PoS terminals, smartphones or computer at retails stores for retrieving information about the item to which it is attached [10], [11]. In fact, barcodes decode fast and most barcodes standards provide redundant information for error correction purpose [12], [13].

There are two types which are linear barcodes and matrix barcodes [12]. Linear barcode is the first generation of barcode ever created and it is in one-dimensional, 1D barcode that is made up from lines and spaces that its own specific pattern. Meanwhile, matrix barcode is also known as $2 \mathrm{D}$ barcode that uses two-dimensional way to present the information [9], which is improved from the one-dimensional barcode that presents data more per unit area.

The 1D barcodes consists of code 39, code 128, EAN-13 and ISBN meanwhile 2D barcodes are such as QR code, PDF417, Data Matrix and Maxi code as shown in Fig. 2.

However, using barcode, identification method is not a good idea because barcode can be reprinted for later usage [14] as illustrated as in Fig. 3.

\begin{tabular}{|c|c|c|c|c|}
\hline \multirow[b]{2}{*}{ 1D barcodes } & Code 39 & Code 128 & EAN-13 & ISBN \\
\hline & & & & \\
\hline & QR Code & PDF417 & DataMatrix & Maxi Code \\
\hline 2D barcodes & 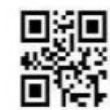 & sisto & & 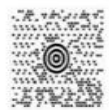 \\
\hline
\end{tabular}

Fig. 2. 1D and 2D Barcodes [9].

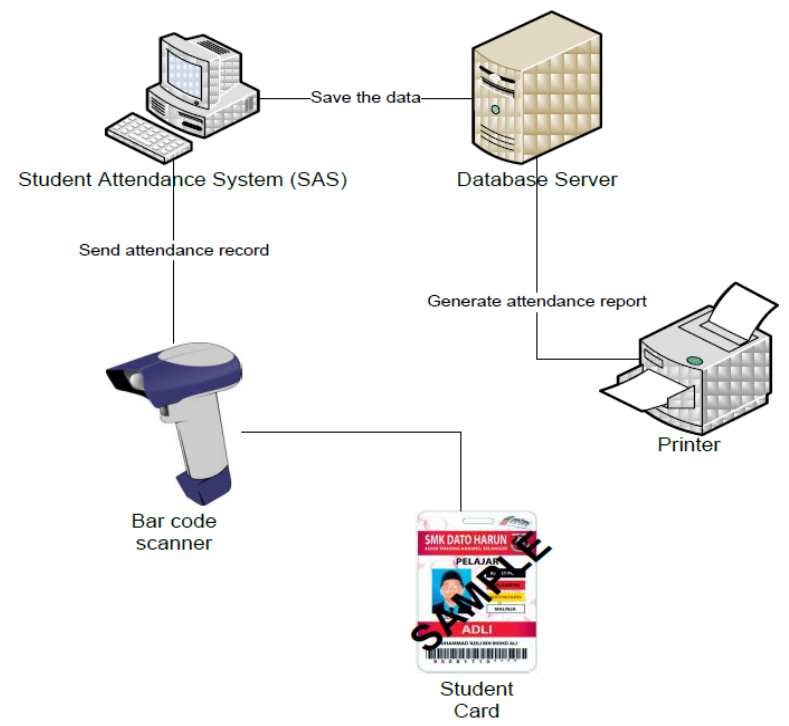

Fig. 3. Barcode System [14].

\section{B. Radio-Frequency Identification (FRID)}

The Radio Frequency Identification (RFID) is a technology that uses a radio waves to automatically identify people or objects from a distance [15] and it is developing drastically [16] in various industries. The RFID technology is designed by developers not only in traditional applications for instance asset or inventory tracking [17], but also in security services such as electronic passports and RFID-embedded credit cards.

Moreover, RFID technology is a non-contact, automatic identification technology to detect, track, sort and distinguish a variety of objects including people, vehicles, goods and assets [18] without the need for direct contact as the magnetic stripe technology or line of sight contact (as found in bar code technology).

RFID technology tracks the activities of objects through a network of radio enabled scanning devices over a several meters of distance. There are three different stages of frequency of RFID in Fig. 4, which is low, high and ultra-high frequency [19] that operated at difference distance.

The RFID system consists of three elements, which is an RFID tag or transponder, RFID tag reader and back-end database which stores record associated with tag information [20] as illustrated in Fig. 5, which each tag contains a unique identity code.

An RFID reader releases a low-level radio frequency magnetic field that energizes the tag. The tag responds to the reader's query and states its presence via radio waves, transmitting its unique identification data. This data is decoded by the reader and passed to the local application system via middleware. The middleware acts as an interface between the reader and the RFID application system. The system examines and verify the identity code with the information stored in the host database [20] or backend system. In this way, accessibility or authorization for further processing can be granted or refused, depending on results received by the reader and processed by the database.

However, current NFC's data privacy is exposed when other user has the same NFC device, which he or she able to read the data using scan random tag number [17]. In order to overcome the data privacy problem, the verification is implemented [12] for better security.

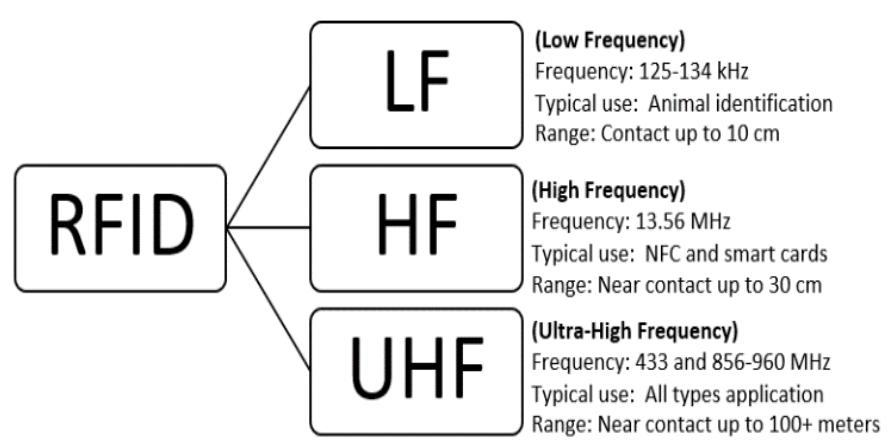

Fig. 4. RFID System [19]. 


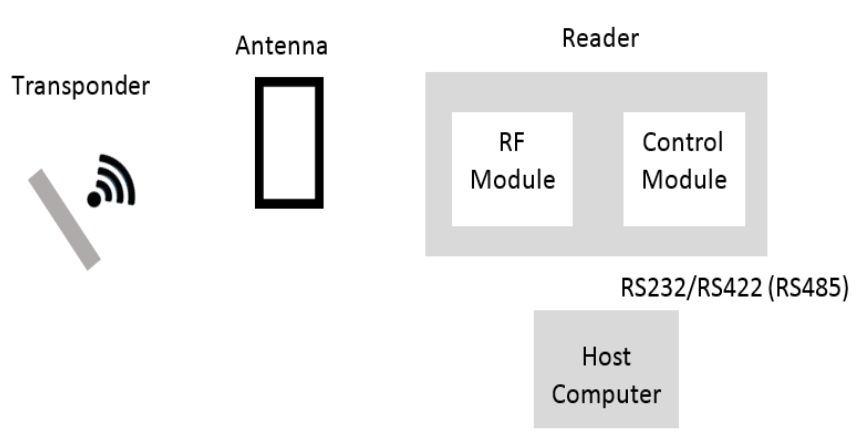

Fig. 5. How RFID Work.

RFID technology increases a number of fears regarding privacy, security and law enforcement. Current RFID's data privacy is exposed when other user has a same NFC device he or she can also read the data and tend to scan random tag number. To overcome data exposure problem, this study is to improve the security of data transmission. Therefore, a new method is proposed for matching the availability of document file which search the information with NFC based on basic system developed. In fact, the new matching method with verification mechanism helps to reduce time when searching the document file at the document room which the employee may not know where the current files are. Furthermore, it is convenient since the process of searching the data is smooth and tend to avoid human mistake that could be stolen or misplace. RFID is chosen since it cannot be easily replicated. It is based on the component content in that RFID which is a small chip that contains its own unique identification and address. Therefore, we use Near Field Communication, NFC technologies to improve its security and make it more systematic.

\section{Near Field Communication (NFC)}

Near Field Communication, NFC is a technology that allows a device to communicate with another hardware at a maximum distance of around less than $10 \mathrm{~cm}$ [21]. NFC is based on RFID that used same working principles, which has interface technology for short-range data communication working in the frequency band of $13.56 \mathrm{MHz}$ [22] with ISO/IEC 18092 standard, compatible to ISO/IEC standards 14443 (proximity cards) and 15693 (vicinity cards) and to Sony's FeliCa contactless smart card system.

A basic principle of the NFC technology is "it's all in a touch". This means that simply touching an object or an NFC device with another NFC device immediately triggers an action. Objects can be equipped with NFC tags. These tags are used to store content like file name, file type or file id number. As shown on Fig. 6, when the NFC based reader tag the NFCtagged that were attached at the item, such as cloth, it shows the detail information about the cloth and the sales assistant has to verify its identity to prove that the customer purchases the cloth.
Thus, NFC can be used with existing infrastructures based on the standards, excluding the need for a separate NFC infrastructure, which is make it easier and more convenient to make transactions, exchange digital content, and connect electronic devices with a touch. Moreover, NFC has been developed between NXP Semiconductors formerly Philips Semiconductors and Sony Corporation since it has the ability to read and write to devices, it is believed that they have a wider use in the future than standard smart cards.

NFC involves an initiator and a target. The initiator, as follows from the name, initiates and actively generates an RF signal and controls the exchange of data where the request is answered by a passive target. The NFC protocol also distinguishes between two modes of communication: active and passive.

Active communication is the initiator and target both communicate by making their own electric fields. The communication is done at half duplex; disengaging their RF field until no other device is transmitting. In this mode, both devices are typically having power supplies.

On the other hand, Passive mode is more common application where the initiator is the only device that generates the RF signal and having a backup key recognition [23]. The presence of the correct key is checked by the wireless link generated between the initiator and key generator. For instance, payment, public parking and toll stations are the application of Passive mode NFC.

Therefore, the proposed system is developed the verification feature to improve the security and the expiry date detection system using NFC. The tag and target device answers that call by modulating the current RF field which the initiator device listens out for, and then processes the signals, later transferring the data. The data rates currently supported are $106,212,424$ or $848 \mathrm{Kbit} / \mathrm{s}$.

The methodology of the proposed system is explained in Section 3.0. Also, section 4.0 demonstrates the results and findings and section 5.0 concludes the study.

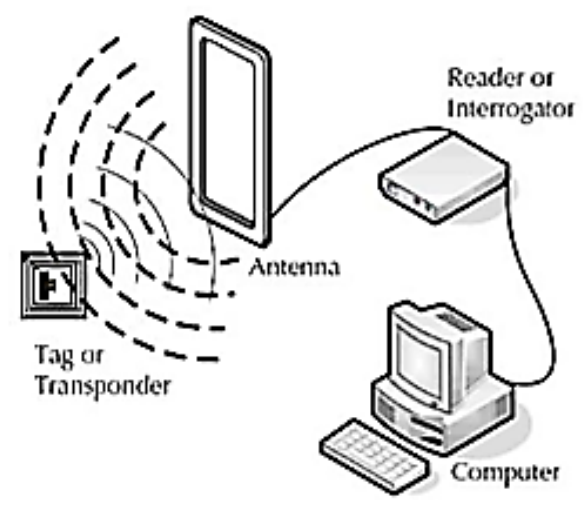

Fig. 6. NFC Topology [20]. 


\section{METHODOLOGY}

The implementation of the document file detection system is using waterfall model as shown in Fig. 7 that consists of six phases, that commonly used in software development and applicable for prototype system.

\section{A. Project Planning and Feasibility Study}

In the phase 1, the project planning is laid out. The feasibility study is implemented to gathered information and requirement such as the objective project is determined, identify and understand what type of current technology used in document file management and determine the suitable software and hardware that planning to use to make the development executed as expected. In this phase, the implementation idea that involves NFC need to be done, functionality and system requirement to accomplish the study. For the hardware specifications, a NFC reader, a smartphone with NFC integrated, a NFC tag and a personal computer is needed for the experiment setup. On the other hand, the software requirements consist of PHPMyAdmin, WAMP server and go to tags for windows are useful for the prototype development.

\section{B. Identify and Analyze Problem}

In the phase 2, the problem is identified and analysed, which is decision to either enhance or propose a method into the physical document management. Moreover, the process to collect data, identify the problem and recommend some suggestions for improving the existing system is also laid out. This phase involves in gathering data, finding solution for overcoming the limitation of the current system and identifying the target users in developing NFC technology. The main objective of this phase is to produce a solution for the proposed system and evaluate other techniques used in other application on data gathering and problem solving.

\section{Propose Network Design}

In phase 3, the requirement and analysis are designed according to topology design, software and hardware selection. Based on the analysis conducted, a new system needs to be proposed and designed. In this phase, planning is designed on network design on physical and logical; and the flow design. Also in this phase, the selection of the software and hardware that going to be use is properly designed. In this phase, we designed the web based application to be developed using personal computer.

\section{Implementation}

In phase 4 shows the installation and configuration of hardware and software which is using NFC and implemented in the real system. A database is created to store the required data and integrated with the NFC reader, NFC tags for the secure system. For the database design, there are two types of database that is conceptual and logical design. Fig. 8 shows the entity relationship diagram (ERD) that refers to the conceptual database. The development of system used the hardware and software required which is NFC reader (hardware), a set of workstation which is PC, CPU, keyboard and mouse (hardware), NFC tag (hardware), GoToTag application (software), PhpMyAdmin (software).

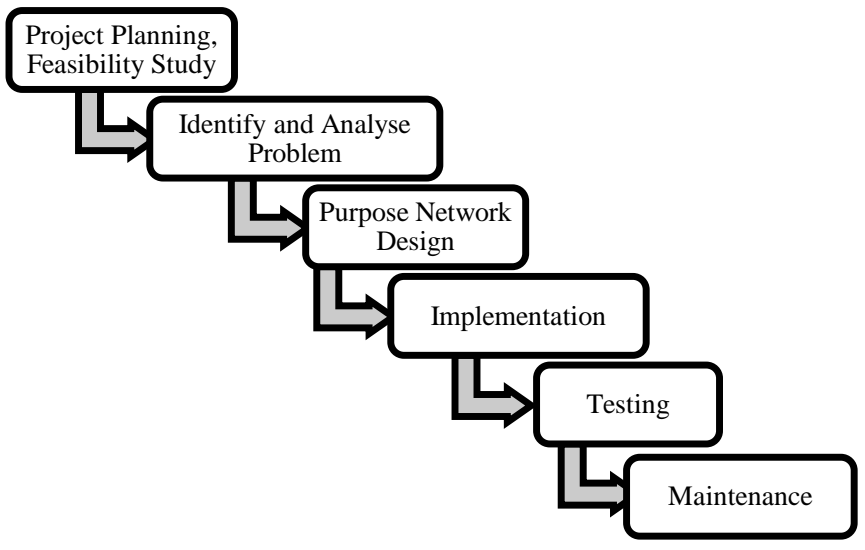

Fig. 7. Waterfall Model Life Cycle.

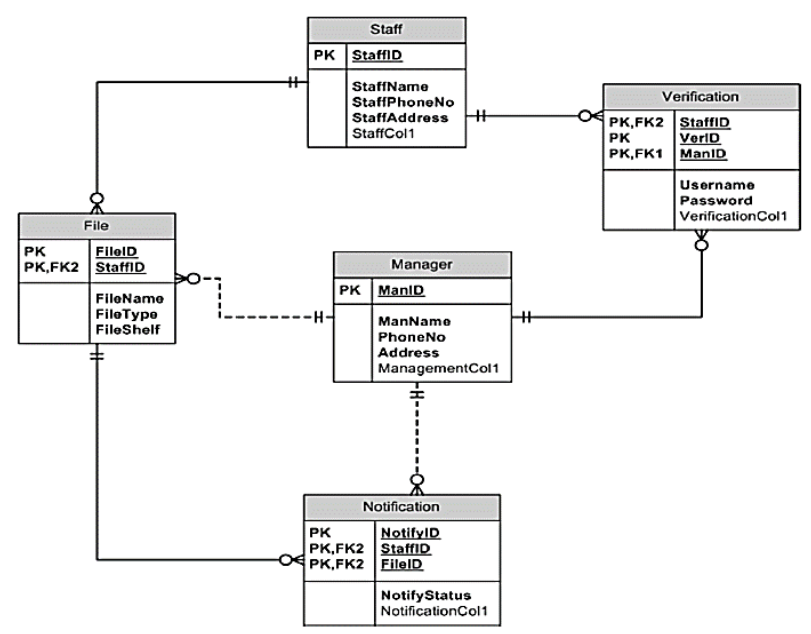

Fig. 8. ERD for the Database Design.

\section{E. Testing}

In phase 5, for testing phase, unit testing, integration testing, and system testing is performed. The unit testing is to ensure that NFC reader is switched on by looking at the green light at the upper right corner of the device and the reader is ready to scan the NFC tag. Besides, the integration testing is determined that the combination of NFC writer, NFC tag, database and developed interfaces are functioned well. At this stage, when the NFC tag is scanned at the NFC reader, then there is a sound of "beep" to indicate that the NFC tag is acknowledged by the NFC reader. After that, the information is appeared on the interfaces showing the details data about the physical files. The system is tested by going through the data validation and error-handling. All testing is to ensure the document file tracking system using NFC environment is working properly.

\section{F. Maintenance}

In phase 6 , the requirement statement need to be accomplished by researchers and any changes need to updated and recorded for future reference. The effectiveness of the system application is based on the level of reliability and validity of a recorded data. The hardware and software is setup as shown in Fig. 9. 

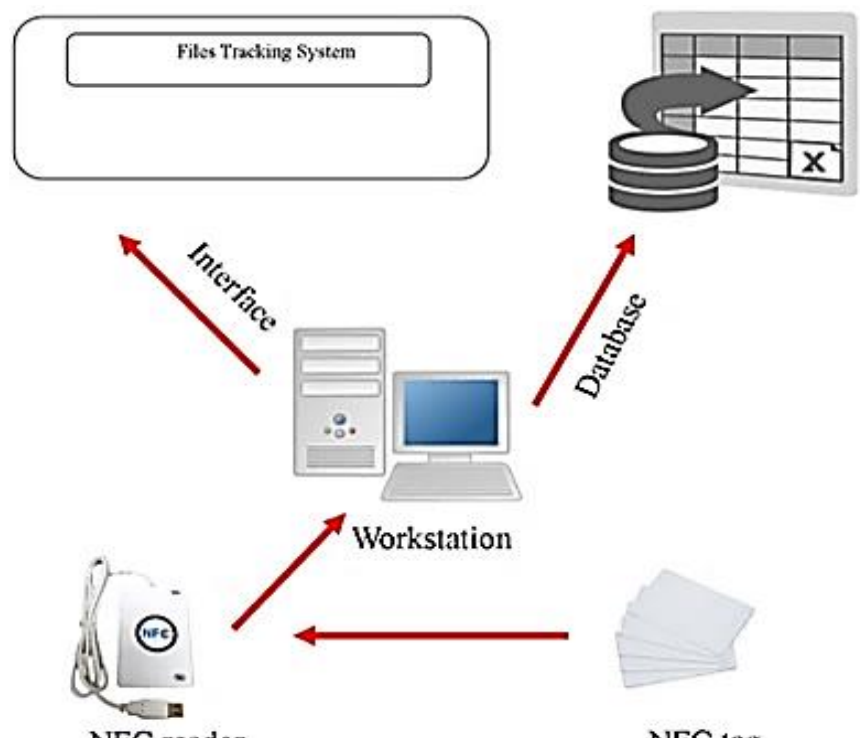

NFC reader
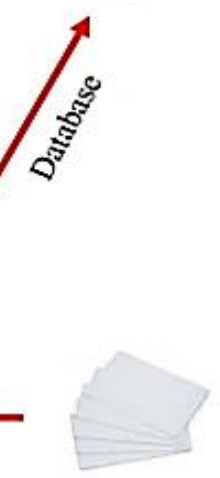

NFC lag

Fig. 9. Hardware and Software Setup of the Proposed System.

\section{RESULTS AND FINDINGS}

In this paper, experiments are shown during the proposed system development, which is the verification and detection of document file detection. The first experiment is to develop the verification feature, which is integrated with databases, Document File Detection System Interfaces and NFC reader as shown as in Fig. 10. The main page displayed that dual verification has occurred when the employee need to scanned his or her ID to the NFC reader to indicate that the employee is the authorized user and the ID number will display on the main page. Then, the NFC tag at the physical document is tapped to scan information about the physical document and the data is appeared on the File ID section by displaying the subject code, semester rack and subject name as in Fig. 11 in order to conform that this physical document belongs to the respective employee.

Then, click the button SAVE to store information into the database. After the data is saved, the pop up menu with "DATA SAVED!" indicates that the data have been safely stored in the database as in Fig. 11. The admin clicked a button "Exit" to end the session.

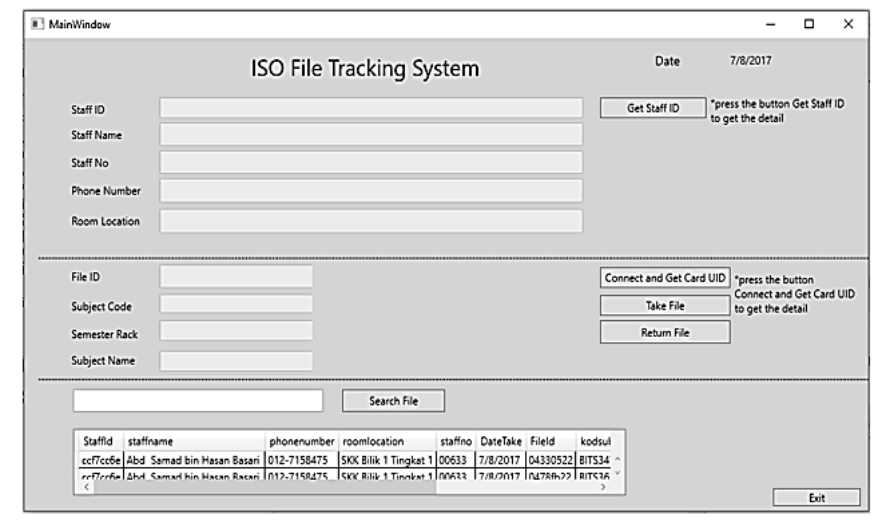

Fig. 10. Main Page.

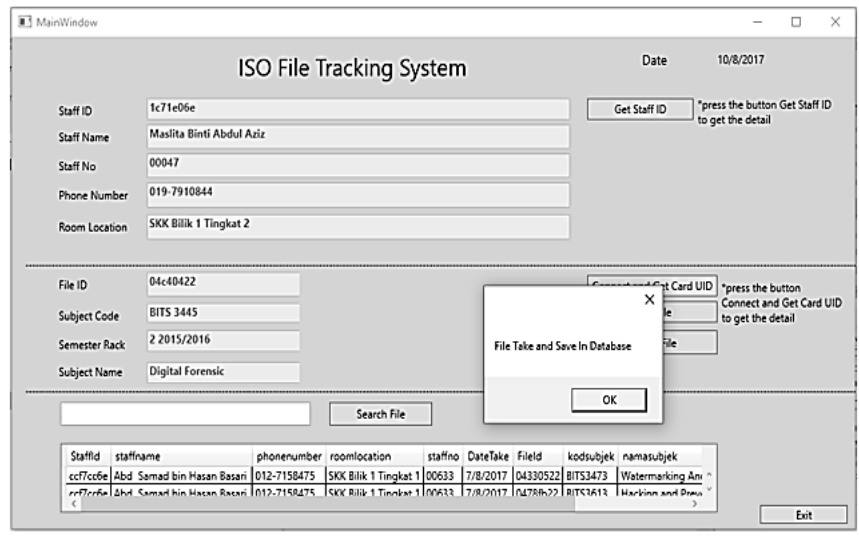

Fig. 11. The Status of Physical Document File.

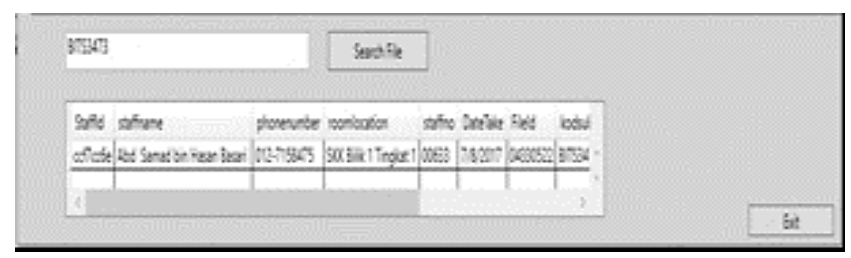

Fig. 12. Physical File Search for Verification.

Fig. 12 shows the user interface as when the user wants to take the physical document out from physical document room, the search file button is used to search the location of the file and the output shows if the physical document is in the room or not. If the physical document is in the room, the information shows the location and employee who currently holds the document file, the information is displayed at the screen.

Based on our findings, employee can view expired or not, which display the items information, the name of the employee who access the system and show the expiry date about the food product from the proposed system.

Another outcome from the findings is the user interface of this proposed system, displayed the list of items tag information with time remaining and status of the current food expiry date, which is operated during the data processing between the NFC tag and the database. This function can be enhanced to be more effective and efficient system with the additional features of automatic read and save data into the database or cloud based database.

The recommendation for future development is there is no need to display the owner of employee's name at record bar because it creates a redundancy with the record displayed at top of the interface and only show the current or latest location of the document file.

\section{CONCLUSION}

In this paper, we proposed a file management detection using NFC (short range) for file management system. The combination of NFC with file management, helps organizations to be more effective and efficient in operation. The proposed system named as Document File Detection integrates security characteristics such as verification feature for making the system more robust and efficient. As a conclusion, the proposed system is recommended to produce a 
better performance and operates in cloud based file tracking system for faster detection. The future system is planning to design that aligned with 4th Industrial Revolution.

\section{ACKNOWLEDGMENT}

Thank you to the Center of Applied Computing Technology (C-ACT), Fakulti Teknologi Maklumat dan Komunikasi (FTMK), Universiti Teknikal Malaysia Melaka (UTeM) and MyRA.

\section{REFERENCES}

[1] Mathioudakis, I. Rousalova, A.A. Gagnat, N. Saad, G. Hardavella, "How to keep good clinical records", Breathe (Sheff), vol. 12 no. 4, pp. 369-373 doi: 10.1183/20734735.018016, 2016.

[2] S.S Zainal Abidin and M.H. Husin, "Improving accessibility and security on document management system: A Malaysian case study", Applied Computing and Informatics, open access journal, DOI: 10.1016/j.aci.2018.04.002, 2018.

[3] A. Husain, M. Bakhtiari and A. Zainal, "Printed Document Integrity Verification using Barcode", Jurnal Teknologi, vol. 70, no. 1, pp. 99106, 2014.

[4] P. Tan, H. Wu, P. Li and H. Xu, "Teaching Management System with Applications of RFID and IoT Technology" Education Sciences, vol. 8, no. 26, pp. 1-13, 2018.

[5] M. Gaspar, J. Julião, M. Cruz, "Organizational Strategies Induced by the Fourth Industrial Revolution: Workforce Awareness and Realignment", Innovation, Engineering and Entrepreneurship. HELIX 2018. Lecture Notes in Electrical Engineering, vol. 505. pp. 330-336, Springer, 2018.

[6] A. Alqarni, M. Alabdulhafith and S. Sampalli, "A Proposed RFID Authentication Protocol based on Two Stages of Authentication", Procedia Computer Science, vol. 37, pp. 503-510, 2014.

[7] U.A. Pozdnyakova, V.V. Golikov, I.A. Peters and I.A. Morozova, "Genesis of the revolutionary transition to industry 4.0 in the $21 \mathrm{st}$ century and overview of previous industrial revolutions. In E. Popkova, Y. Ragulina and A. Bogoviz A. (eds) Industry 4.0: Industrial Revolution of the 21st Century", Springer, vol. 169, pp.11-19, 2019.

[8] M. Akshatha, K.P. Alankrutha, A. G. Janitha, M. Lavita, V. G. Smitha, "Student Authentication and Verification System using Barcode Scanner", International Journal of Internet of Things, vol. 6, no. 2, pp. 71-74, 2017.

[9] A. Shaikh, S. Mali, S. Tambe and A. Yadav, "Mobile barcode system with inventory management and employee work tracking using Google Analytics", International Journal of Engineering and Computer Science, vol. 4, no. 2, pp. 10547-10549, 2015.

[10] M. Gangurde, P. Ghule, P. Kasar, A. Satpute and S. Tupe, "Business
Logistics System by using Robust Barcode Scanner", Imperial Journal of Interdisciplinary Research, vol. 3, no. 4, pp. 1528-1530, 2017.

[11] N.M.Z. Hashim, N. Ibrahim, N.M Saad, F. Sakaguchi and Z. Zakaria "Barcode Recognition System", International Journal of Emerging Trends \& Technology in Computer Science, vol. 2, no. 4, pp. 278-283, 2013.

[12] [12] P. Bodnar, \& L.G. Nyul. (2012). Improving Barcode Detection with Combination of Simple Detectors. [Online]. Available FTP: https://doi.org/10.1109/SITIS.2012.52.

[13] H. Subramaniam, M. Hassan, and S. Widyarto. "BarCode Scanner Based Student Attendance System (SAS)", Journal of Technology of Information and Communication (Jurnal TIKOM), vol. 1, no.3, pp. 173177, 2013.

[14] S.W. Park and I.Y. Lee, "Anonymous Authentication Scheme Based on NTRU for the Protection of Payment Information in NFC Mobile Environment", Journal of Information Process System, vol. 9, pp. 461476, 2013.

[15] D. Parkash, T. Kundu and P. Kaur, "The RFID Technology and Its Applications: A Review", International Journal of Electronics, Communication and Instrumentation Engineering Research and Development, vol. 2, no. 3, pp. 109-120, 2012.

[16] G. Arcese, G. Campagna, S. Flammini and O. Martucci,", Near-field Communication: Technology and Market Trends", Technologies, vol.2, pp. 143-163, 2014.A. Yewatkar, F. Inamdar,

[17] R. Singh, A. Ayushya and A. Bandal, "Smart Cart with Automatic Billing, Product Information, Product Recommendation Using RFID \& Zigbee with Anti-Theft", Procedia Computer Science, vol. 79, pp. 793800, 2016.

[18] D.B. Datta. "Radio Frequency Identification Technology: An Overview of its Components, Principles and Applications", International Journal of Science, Engineering and Technology Research, vol. 5, no. 2, pp. 565574, 2016.

[19] Atlas RFID Store. (2012). The basics of an RFID system, [Online]. Available FTP: http://rfid.atlasrfidstore.com/hs-fs/hub/300870/file252314647pdf/Content/basics-of-an-rfid-system-atlasrfidstore.pdf.

[20] K. Curran, A. Millar and C.M. Garvey, "Near Field Communication", International Journal of Electrical and Computer Engineering, vol. 2, no 3, pp. 371-382, 2012.

[21] W. Suparta, "Application of Near Field Communication Technology for Mobile Airline Ticketing", Journal of Computer Science, vol. 8, no. 8, pp. 1235-1243, 2012.

[22] G. Arcese, G. Campagna, S. Flammini and O. Martucci, "Near Field Communication: Technology and Market Trends", Technologies, vol.2, pp. 143-163, 2014.

[23] R. Steffen, J. Preißinger, T. Schöllermann, A. Müller and I. Schnabel, "Near Field Communication in an Automotive Environment use cases, architecture and realization", Second International Workshop on IEEE, pp. 15-20, 2010. 